\title{
Improved outcome for AML patients over the years 2000-2014
}

Sarah Bertoli ${ }^{1,2,3}$, Suzanne Tavitian ${ }^{1}$, Anne Huynh ${ }^{1}$, Cécile Borel ${ }^{1}$, Sarah Guenounou ${ }^{1}$, Isabelle Luquet ${ }^{4}$, Eric Delabesse ${ }^{4}$, Audrey Sarry ${ }^{1}$, Guy Laurent ${ }^{1,2}$, Michel Attal ${ }^{1,2}$, Françoise Huguet ${ }^{1}$, Emilie Bérard ${ }^{5,6}$ and Christian Récher ${ }^{1,2,3}$

\begin{abstract}
Few recent studies from registries have reported an improvement in overall survival of younger patients with acute myeloid leukemia (AML). However, reasons for this improvement are not defined. We analyzed the therapeutic course and outcome of 976 patients treated by intensive chemotherapy between 2000 and 2014. The number of patients receiving allogeneic stem cell transplantation in first or second response significantly increased over time whereas autologous transplantation was progressively abandoned. In the 513 younger patients, there were no differences in first complete response, induction failure, incidence of relapse, or non-relapse mortality over time. The period of time was significantly associated with a better overall survival especially in 2010-2014. The 2010-2014 period effect was still significant in multivariate analysis and was independent of allogeneic stem cell transplantation. In the 463 older patients, there was a significant interaction between the period and leukocytosis in multivariate analysis meaning that the 2010-2014 period had only an impact in patients with white blood cell count $>50$ giga/L for response and overall survival. Progresses have been made in each phase of the therapeutic course of younger AML patients resulting in survival improvement. In older patients, the outcome of hyperleukocytic patients has significantly improved in 2010-2014.
\end{abstract}

\section{Introduction}

Acute myeloid leukemia (AML) is a hematological malignancy in which no key specific treatment has been approved compared to the progress made with multiple myeloma, B-cell lymphoma, chronic myelocytic leukemia, or acute lymphoblastic leukemia, which all recently benefited from the registration of very potent drugs ${ }^{1,2}$. As far as younger and fit older AML patients are concerned, the therapeutic strategy has remained unchanged for decades based on the so called " $7+3$ " induction chemotherapy using continuous-infusion cytarabine with an anthracycline. In patients having achieved complete remission, standard post remission treatments include chemotherapy as well as hematopoietic stem cell

\footnotetext{
Correspondence: Christian Récher (recher.christian@iuct-oncopole.fr)

'Service d'Hématologie, Centre Hospitalier Universitaire de Toulouse, Institut Universitaire du Cancer de Toulouse Oncopole, Toulouse, France

${ }^{2}$ Université Toulouse III Paul Sabatier, Toulouse, France

Full list of author information is available at the end of the article

Sarah Bertoli and Suzanne Tavitian contributed equally to this work.
}

transplantation. Decision making for allogeneic transplantation relies on the probability of relapse, better predicted by the AML genetic risk and on the risk of treatment-related death predicted by specific scorings ${ }^{3}$.

Yet, few recent studies from registries or compilation of clinical trials have reported an improvement in overall survival (OS) of adult AML patients, especially in patients of 60 years of age or younger ${ }^{4-8}$. Population-based studies from registries can provide a more complete assessment of progress in survival than is available from clinical trials, which often preselect younger and generally healthier subjects and may not be representative of the average diagnosed individual. Studying survival trends over time can provide information about cancer treatment and control efforts. However, though these studies deal with large numbers, variable provided remains scarce (i.e., age, sex, and survival) and reasons for this improvement are not clearly defined. Such reasons for improvement are likely to be multiple as the road to cure for AML patients is a multi-step process subjected to numerous events and 
adverse effects, as well as various treatment options, especially with regards to allogeneic stem cell transplantation allocation or access to clinical trials. Although there has been no improvement in " $7+3$ " induction using a third drug to treat AML, despite many attempts, many other key points of the treatments have evolved over time in current practice especially with respects to supportive care, stratification of treatment according to genetic risk and allogeneic stem cell transplantation indications.

We analyzed the main changes in routine practice and the outcome of AML patients treated between 2000 and 2014 by intensive chemotherapy in order to determine whether there has been an improvement in survival over time and if this improvement is independent of standard prognostic factors.

\section{Subjects and methods Patients}

Between January 2000 and December 2014, a total of 976 patients with a newly diagnosed AML were consecutively treated with intensive chemotherapy at Toulouse University Hospital. The leukemia unit of the Toulouse University Hospital is the only certified center for the treatment of AML in the Midi-Pyrénées region (Southwest of France), which contains 3 million inhabitants. Patients are referred by personal physicians or primary care centers and are firstly seen by leukemia specialists either as outpatients for rapid diagnosis and work-up or directly as in patients if urgent medical interventions are needed. Patients from the Midi-Pyrénées area are recorded each week in the leukemia unit according to guidelines from the Oncomip network (http://www.oncomip.org) after informed consent. Patients were included in this study if they received at least one dose of intensive chemotherapy regimen. Patients with acute promyelocytic leukemia were not considered. The cytogenetic classification was in accordance with the Medical Research Council (MRC) classification ${ }^{9}$. Molecular analyses were performed at diagnosis or retrospectively from samples stored in the tumor bank of the U1037 Inserm department (no. DC-2008-307CPTP1 HIMIP) ${ }^{10}$. Data were collected from the patients' files and certified by the Data Management Committee of the anonymized AML database of Toulouse University Hospital and registered at the Commission Nationale de l'Informatique et des Libertés (CNIL) under access No. 1778920. In accordance with the Declaration of Helsinki, the study was reviewed and approved by the research ethics committee at Toulouse University Hospital.

\section{Treatments}

Patients received intensive induction chemotherapy that included daunorubicin or idarubicin together with cytarabine either by continuous intravenous or bolus infusion as part of, or according to French clinical trials for younger and older ( $>60$ years) AML patients ${ }^{11-16}$. Hydroxyurea could be given promptly at diagnosis for white blood cell (WBC) reduction in hyperleukocytic patients. Leukapheresis were not performed in our institution. Responding patients with intermediate or high risk AML and a matched sibling or 10/10 HLA allele fully matched unrelated donor were allocated to allogeneic stem cell transplantation. Patients with no HLA-matched donor received a consolidation regimen consisted of highdose cytarabine followed by autologous stem-cell transplantation or three courses of high-dose cytarabine. After achieving complete response (CR), patients over 60 received maintenance therapy with idarubicin and lowdose cytarabine. Refractory or relapsed patients could receive salvage therapy using several chemotherapy regimens of different dose-intensity that are described in Supplementary Methods. Supportive care that included treatment of febrile neutropenia and disseminated intravascular coagulopathy, as well as blood-product transfusions, were given according to guidelines, which did not change over the study period and were in accordance with standard recommendations ${ }^{17}$. CR referred to the combination of $C R$ and $C R$ with incomplete blood count recovery $(\mathrm{CRi})$ after one or two induction cycles were defined by the international consensus criteria ${ }^{17}$.

Conditioning regimens for allogeneic stem cell transplantation were classified according to a consensus definition of conditioning regimen intensity ${ }^{18}$. Myeloablative conditioning consisted of high-dose cyclophosphamide with total-body irradiation or busulfan. Reduced-intensity and non-myeloablative conditioning were grouped together under the reduced-intensity conditioning which associated busulfan, fludarabine and antithymocyte globulin or fludarabine and low total-body irradiation. Graft vs. host disease prevention consisted in cyclosporine alone or in combination with either methotrexate or mycophenolate mofetil. Conditioning regimens for autologous stem cell transplantation have been published elsewhere $^{19}$.

\section{Outcomes}

Primary outcome was OS. Secondary outcomes were day-60 death (to the date of diagnosis), first and second $\mathrm{CR}$, induction failure, cumulative incidence of relapse (CIR), non-relapse mortality (NRM), and disease-free survival (DFS $)^{17}$. CIR was measured only for patients achieving CR, from the date of remission achievement to the date of relapse. NRM was treated as competing events of relapse and patients not known to have died at last follow-up are censored on the date they were last known to be alive. DFS was measured only for patients achieving $\mathrm{CR}$, from the date of remission achievement to the date of relapse or death from any cause; patients not known to 
have died at last follow-up are censored on the date they were last known to be alive.

\section{Statistical analyses}

Since therapeutic strategies of AML differed between younger and older patients, we analyzed separately the outcome of patients $<60$ years $(n=513)$ and patients aged 60 years or older $(n=463)$ during the 2000-2004, 2005-2009, and 2010-2014 periods. We first described patients' characteristics at diagnosis using number and frequency for qualitative data; median and interquartile range (IQR) for quantitative data. Differences in day-60 death, response, and induction failure according to the periods were tested in univariate analyses using $\chi^{2}$ test (or Fisher's exact test in case of small expected numbers). Multivariate analyses were conducted using logistic regression. For univariate survival analyses of OS and DFS, Kaplan-Meier survival curves were drawn and differences in survival functions were tested using the LogRank test. Univariate survival analyses used Cumulative Incidence Functions and Gray's test for CIR and NRM, since NRM and relapse were treated as competing events. Adjusted hazard ratios (HR) and 95\% confidence intervals (CI) were assessed using a standard Cox model, for OS and DFS, and a proportional sub distribution hazard model which is an extension of the Cox model for the situation of competing risks, for CIR and $\mathrm{NRM}^{20}$. Multivariate analyses initially included periods together with potential confounding factors (age, WBC count, AML status, cytogenetic risk, and allogeneic stem-cell transplantation for OS, DFS, and CIR). Then we used stepwise regression to assess variables that were significantly and independently associated with endpoints $(P<0.05)$. The proportional-hazard assumption was tested for each covariate of the Cox model by the "log-log" plot method curves and was always met. When linearity hypothesis was not respected, continuous potential confounding factors were transformed into ordered data. Interactions between the period and independent covariates were tested in final models. Allogeneic stem-cell transplantation was evaluated as time-dependent covariate. In order to compare patients with homogeneous maximal follow-up, patients in the first and second period were censored at 7 years. All reported $P$-values were two-sided and the significance threshold was $<0.05$. Statistical analyses were performed on STATA ${ }^{\circ}$ version 14.1 (STATA Corp., College Station, TX).

\section{Results}

Main changes in routine practice during the study period

Chemotherapy regimens used in younger patients $(<60$ years) during the study period, as well as doses of anthracyclines and cytarabine, are shown in Supplementary Table 1. Anthracyclines were no longer used in consolidation from 2010. Although anthracycline doseintensification during induction with either daunorubicin $\left(270 \mathrm{mg} / \mathrm{m}^{2}\right)$ or idarubicin $\left(45 \mathrm{mg} / \mathrm{m}^{2}\right)$ started as soon as 2010, the cumulative dose of daunorubicin or idarubicin was lower in the 2010-2014 period. In older patients ( $\geq 60$ years), idarubicin and cytarabine doses did not change during the study period at both induction and consolidation ${ }^{21}$. With regards to prophylaxis of invasive fungal infections during induction chemotherapy, fluconazole was used in the earlier period followed by voriconazole or caspofungin in 2003 and then, posaconazole in $2008^{22,23}$. Starting from 2010 , dexamethasone $(10 \mathrm{mg}$ b.i.d, 3 days) was added to induction chemotherapy in patients with $\mathrm{WBC}>100 \mathrm{giga} / \mathrm{L}$ or in patients with $>50$ giga/L and symptoms of leukostasis ${ }^{24}$. Indications for allogeneic stem cell transplantation in first CR evolved from geno-identical to pheno-identical (from 2007) and anecdotally umbilical cord blood (from 2005), whereas autologous stem cell transplantation was progressively abandoned. In older patients, the first allogeneic stem cell transplantation with reduced-intensity regimen was performed in 2002 in patients of 50-60 years and in 2007 in patients older than 60 . Molecular stratification for allogeneic stem cell transplantation indications based on NPM1, FLT3-ITD, and CEBPA mutations in patients with intermediate-cytogenetic risk was introduced in 2006. From this date, patients with favorable molecular risk (i.e., mutation of NPM1 or CEBPA without FLT3-ITD mutation) were no longer allocated to allogeneic transplantation in first $\mathrm{CR}$ and received three cycles of high-dose cytarabine as post remission therapy or an autologous transplantation $^{25}$. A specific unit dedicated to acute leukemia was created in the Hematology department in 2006.

\section{Outcome of the whole cohort}

Outcome of the whole population stratified on main prognostic factors (cytogenetics, de novo vs. secondary or therapy-related AML, mutational status) during to the 2000-2004, 2005-2009, and 2010-2014 periods are shown in Supplementary Fig. 1. However, since therapeutic strategies differed between younger and older patients, we analyzed separately the outcome of patients $<60$ years and patients $\geq 60$ years during the three periods.

\section{Outcome of younger ( $<60$ years) patients}

The characteristics of the 513 younger patients including age, AML status (de novo vs. secondary), performance status, WBC count, cytogenetic risk and FLT3-ITD, NPM1, or CEBPA mutations in intermediate-cytogenetic risk were well balanced during the study period (Table 1). Median follow-up of patients still alive was 67.4 months (84.0, 74.1, and 38.1 months for 2000-2004, 2005-2009, and 2010-2014, respectively). Table 2 shows response to induction, treatment distribution and main outcomes. 
Table 1 Characteristics of younger ( $<60$ years) AML patients

\begin{tabular}{|c|c|c|c|c|}
\hline & $\begin{array}{l}2000-2004 \\
N=173 \\
(33.7 \%)\end{array}$ & $\begin{array}{l}2005-2009 \\
N=181 \\
(35.3 \%)\end{array}$ & $\begin{array}{l}2010-2014 \\
N=159 \\
(31.0 \%)\end{array}$ & $\begin{array}{l}\text { Total } \\
\mathrm{N}=513 \\
(100 \%)\end{array}$ \\
\hline \multicolumn{5}{|l|}{ Follow-up ${ }^{a}$} \\
\hline Median, months (IQR) & $84.0(84.0-84.0)$ & $74.1(61.3-84.0)$ & $38.1(26.4-54.4)$ & $67.4(42.9-84)$ \\
\hline Male, $n(\%)$ & $94(54.3)$ & $86(47.5)$ & $86(54.1)$ & $266(51.9)$ \\
\hline $\begin{array}{l}\text { Female, } n(\%) \\
\text { Age, years (y) }\end{array}$ & \multicolumn{4}{|c|}{ Age, years (y) } \\
\hline Median (IQR) & $47.9(36.4-54.5)$ & $47.3(37.3-55.6)$ & $50.5(35.7-55.7)$ & $48.1(36.6-55.0)$ \\
\hline$<50$ years, $n(\%)$ & $103(59.5)$ & $104(57.5)$ & $78(49.1)$ & $285(55.6)$ \\
\hline$\geq 50$ years, $n(\%)$ & $70(40.5)$ & $77(42.5)$ & $81(50.9)$ & $228(44.4)$ \\
\hline \multicolumn{5}{|l|}{ AML status, $n(\%)$} \\
\hline De novo & $139(80.3)$ & $148(81.8)$ & $134(84.3)$ & $421(82.1)$ \\
\hline Secondary & $34(19.7)$ & $33(18.2)$ & $25(15.7)$ & $92(17.9)$ \\
\hline \multicolumn{5}{|c|}{ Performance status, $n(\%)$} \\
\hline $0-1$ & $95(77.2)$ & $128(89.5)$ & $113(75.8)$ & $336(81.0)$ \\
\hline $2-4$ & $28(22.8)$ & $15(10.5)$ & $36(24.2)$ & $79(19.0)$ \\
\hline \multicolumn{5}{|l|}{ WBC, (giga/L) } \\
\hline Median, IQR & $11(3.3-46.3)$ & $13.3(3.3-41)$ & $9(2.7-45.8)$ & $10.8(3.1-43.5)$ \\
\hline$\leq 50, n(\%)$ & $130(75.6)$ & $145(80.1)$ & $121(76.1)$ & $396(77.3)$ \\
\hline$>50, n(\%)$ & $42(24.4)$ & $36(19.9)$ & $38(23.9)$ & $116(22.7)$ \\
\hline \multicolumn{5}{|l|}{ Cytogenetic risk, $n(\%)$} \\
\hline Favorable & $24(14.0)$ & $28(15.5)$ & $18(11.5)$ & $70(13.7)$ \\
\hline Intermediate & $104(60.5)$ & $112(61.9)$ & $106(67.5)$ & $322(63.1)$ \\
\hline Adverse & $44(25.6)$ & $41(22.7)$ & $33(21)$ & $118(23.1)$ \\
\hline \multicolumn{5}{|c|}{ FLT3-ITD mutation, $n(\%)$} \\
\hline No & $87(76.3)$ & $107(79.3)$ & $101(77.1)$ & $295(77.6)$ \\
\hline Yes & $27(23.7)$ & $28(20.7)$ & $30(22.9)$ & $85(22.4)$ \\
\hline \multicolumn{5}{|l|}{ NPM1 mutation, $n(\%)$} \\
\hline No & $74(75.5)$ & $81(66.4)$ & $88(66.7)$ & $243(69)$ \\
\hline Yes & $24(24.5)$ & $41(33.6)$ & $44(33.3)$ & $109(31)$ \\
\hline \multicolumn{5}{|l|}{ CEBPA mutation, $n(\%)^{\mathrm{b}}$} \\
\hline No & $65(83.3)$ & $72(88.9)$ & $74(90.2)$ & $211(87.6)$ \\
\hline Yes & $13(16.7)$ & $9(11.1)$ & $44(9.8)$ & $30(12.4)$ \\
\hline
\end{tabular}

IQR interquartile range, WBC white blood cell count

${ }^{a}$ Of non-deceased patients

${ }^{b}$ According to ELN 2010 classification

During the induction phase, the 60-day death rate was reduced from $9.2 \%$ in $2000-2004$ to $4.4 \%$ in $2010-2014$ ( $P$ $=0.207$ ) whereas $C R$ and induction failure rates were similar across the study periods. In the post remission phase, the number of patients receiving allogeneic stem cell transplantation gradually increased in a significant manner $(30.5$ vs. $38.5 \%$ vs. $51.8 \%$ in $2000-2004$, 2005-2009, and 2010-2014 periods, respectively; $P=$ $0.001)$. This holds true for relapsed patients who achieved a second CR $(P<0.001)$. By contrast, the rate of autologous stem cell transplantation dramatically decreased (29.1 vs. $2.2 \%$ in $2000-2004$ and $2010-2014$ periods, respectively; $P<0.001)$. In the whole cohort of patients in first $C R$, the cumulative incidence of death without relapse (i.e., NRM) was $12 \%(95 \% \mathrm{CI}, 0.09-0.15)$ and was not significantly associated with the period $(P=0.659)$ in univariate analysis (Fig. 1). The cumulative incidence of death without relapse in patients allografted in first CR $(n$ $=171)$ was $20 \%(95 \% \mathrm{CI}, 0.14-0.27)$ and was not significantly associated with the period $(P=0.940)$. The CIR was $44 \%(95 \% \mathrm{CI}, 39-49)$ with a trend to decrease over time (HR 0.89, 95\% CI, 0.63-1.26; $P=0.498$ and HR 0.78, 95\% CI, $0.54-1.11 ; \quad P=0.169$, for 2005-2009 and 2010-2014 periods as compared to 2000-2004, respectively) (Fig. 1). Multivariate analyses with regards to 60day induction death (HR 0.43, 95\% CI, 0.17-1.13; $P=$ 0.089 ) and DFS (HR 0.76, 95\% CI 0.54-1.06; $P=0.104$ ) showed a trend for a better outcome in the 2010-2014 period than in 2000-2004 (Supplementary Tables 2 and 3). Univariate analysis showed that the period of time was significantly associated with a better OS $(P=0.031)$ with HR of 0.92 (95\% CI $0.70-1.20 ; P=0.536)$ and 0.68 (95\% CI, 0.50-0.92; $P=0.012$ ) for 2005-2009 and 2010-2014, respectively, compared to 2000-2004 (Fig. 1). The 2010-2014 period effect was still significant in multivariate analysis after adjustment for age ( $<$ vs. $\geq 50$ years), AML status (de novo vs. secondary), cytogenetics, WBC ( $\leq$ vs. $>50$ giga/L) and allogeneic stem cell transplantation in CR1 (HR 0.66, 95\% CI 0.49-0.91; $P=0.011$ ) (Table 3). There was no significant interaction between the study period and the other independent variables in the final model indicating that the period effect was not significantly different according to WBC count, AML status, age, cytogenetic risk, or allogeneic stem cell transplantation in CR1. However, to illustrate the impact of the study period according to cytogenetics risk, WBC count, age ( $<$ vs. $\geq 50$ years), and AML status (de novo vs. secondary), Kaplan-Meier survival curves are shown in Supplementary Fig. 2 A-D.

\section{Outcome of older ( $\geq 60$ years) patients}

The characteristics of the 463 older patients were well balanced during the study period (Supplementary Table 4). Median follow-up of patients still alive was 52.5 months (84.0, 70.6, and 35.6 months for 2000-2004, 2005-2009, and 2010-2014, respectively). Table 4 shows response to induction, treatment distribution and main outcomes. During the induction phase, the 60-day death rate was reduced from $15.8 \%$ in $2000-2004$ to $10.2 \%$ in 2010-2014 $(P=0.196)$. This trend was also observed in 
Table 2 Outcome of younger (<60 years) AML patients

\begin{tabular}{|c|c|c|c|c|}
\hline & $2000-2004$ & 2005-2009 & 2010-2014 & $\mathbf{P}$ \\
\hline & $N=173$ & $N=181$ & $N=159$ & \\
\hline Day-60 death, $n(\%)$ & $16(9.2)$ & $15(8.3)$ & $7(4.4)$ & 0.207 \\
\hline CR1, n (\%) & $141(81.5)$ & $148(81.8)$ & $137(86.2)$ & 0.449 \\
\hline Induction failure, $n(\%)$ & $18(10.4)$ & $22(12.2)$ & $14(8.8)$ & 0.603 \\
\hline Allo-SCT in CR1, $n(\%)$ & $43(30.5)$ & $57(38.5)$ & $71(51.8)$ & 0.001 \\
\hline Auto-SCT in CR1, $n$ (\%) & $41(29.1)$ & $20(13.5)$ & $3(2.2)$ & $<0.001$ \\
\hline CR2, n (\%) & $20 / 68^{\mathrm{a}}(29.4)$ & $30 / 60^{\mathrm{a}}(50.0)$ & $22 / 47^{\mathrm{a}}(46.8)$ & 0.040 \\
\hline Allo-SCT in CR2, $n(\%)$ & $0(0)$ & $1(3.3)$ & $9(40.9)$ & $<0.001$ \\
\hline Clinical trial, $n(\%)$ & $98(56.6)$ & $46(25.4)$ & $49(30.8)$ & $<0.001$ \\
\hline Median DFS, months (IQR) & 25.1 (8.3-NR) & 23.5 (7.5-NR) & 43.4 (11.9-NR) & 0.381 \\
\hline Median OS, months (IQR) & 24.4 (8.7-NR) & 26.6 (9.1-NR) & NR (13.4-NR) & 0.035 \\
\hline
\end{tabular}

$C R 1$ first complete response, $C R 2$ second complete response, DFS disease-free survival, IQR interquartile range, OS overall survival, SCT stem cell transplantation

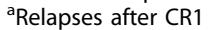

multivariate analysis without reaching statistical significance (OR 0.61, 95\% CI 0.30-1.24; $P=0.173$ ) (Supplementary Table 5$)$. There was a trend for a higher CR rate in the $2010-2014$ period ( $74.9 \%$ vs. 66.9 and $65.6 \%$ in 2000-2004 and 2005-2009, respectively). In multivariate analysis, there was a significant interaction between the period and the WBC count indicating a significant period effect only in patients with $\mathrm{WBC}>50$ giga/L. Indeed, the probability to reach CR was significantly higher in these patients in the 2010-2014 period compared with 2000-2004 (OR 3.90, 95\% CI 1.30-11.7; $P=0.015$ ) (Table 5). Similar to younger patients, the number of patients receiving allogeneic stem cell transplantation significantly increased over time in both first and second CRs (Table 4). In patients reaching first CR, the cumulative incidence of death without relapse was 14\% (95\% CI, 10-18) and was not significantly associated with the period $(P=0.700)$. The CIR was $68 \%(95 \% \mathrm{CI}, 62-73)$ and not associated with the period $(P=0.980)$ even after adjustment (Supplementary Table 6). There was no difference in DFS (Table 4 and Supplementary Table 6) and OS over time (Fig. 2a). Finally, there was a significant interaction between the period and the WBC count in the multivariate analysis for OS meaning that the 2010-2014 period had an impact only in patients with $\mathrm{WBC}>$ 50 giga/L independently of allogeneic stem cell transplantation (HR 0.41, 95\% CI $0.24-0.71 ; \quad P=0.002$ ) (Table 6 and Fig. 2b-c). There was no significant interaction between the study period and the other independent variables in the final model indicating that the period effect was not significantly different according to AML status, age, or cytogenetic risk. However, to illustrate the impact of the study period according to cytogenetics risk, age ( $<$ vs. $\geq 70$ years) and AML status (de novo vs. secondary), Kaplan-Meier survival curves are shown in Supplementary Fig. 3 A-C.

\section{Discussion}

This analytic study shows that the survival of younger adult patients with AML and at least a subset of older patients, has improved in the recent period. This improvement is likely to be multifactorial. Indeed, progresses have been observed in each phase of the therapeutic course in younger AML patients, including less early deaths during induction, more allogeneic stem cell transplantation without increased NRM and more second remissions. It is plausible that the combination of all these factors resulted in survival improvement although it remains challenging to weight the impact of each phase of the treatment course. It can be easily speculated that the significant increase of allogeneic stem cell transplantations could have the higher impact in survival improvement $^{26,27}$. However, the period effect was independent of allogeneic stem cell transplantation. In older patients, significant advances remain to be made although the outcome of hyperleukocytic patients has been significantly improved.

With regards to chemotherapy regimen used over time, although the anthracycline dose intensity has been increased in 2010-2014 during induction by using daunorubicin at a dose of $90 \mathrm{mg} / \mathrm{m}^{2}$ (3 days) or idarubicin at a dose of $9 \mathrm{mg} / \mathrm{m}^{2}$ (5 days), the cumulative dose of both drugs was higher in the earlier period of the study. Indeed, we stopped using anthracyclines during consolidation from $2010^{28,29}$. This suggests that both dose-intensity and timing of administration (e.g., the highest dose early in the 


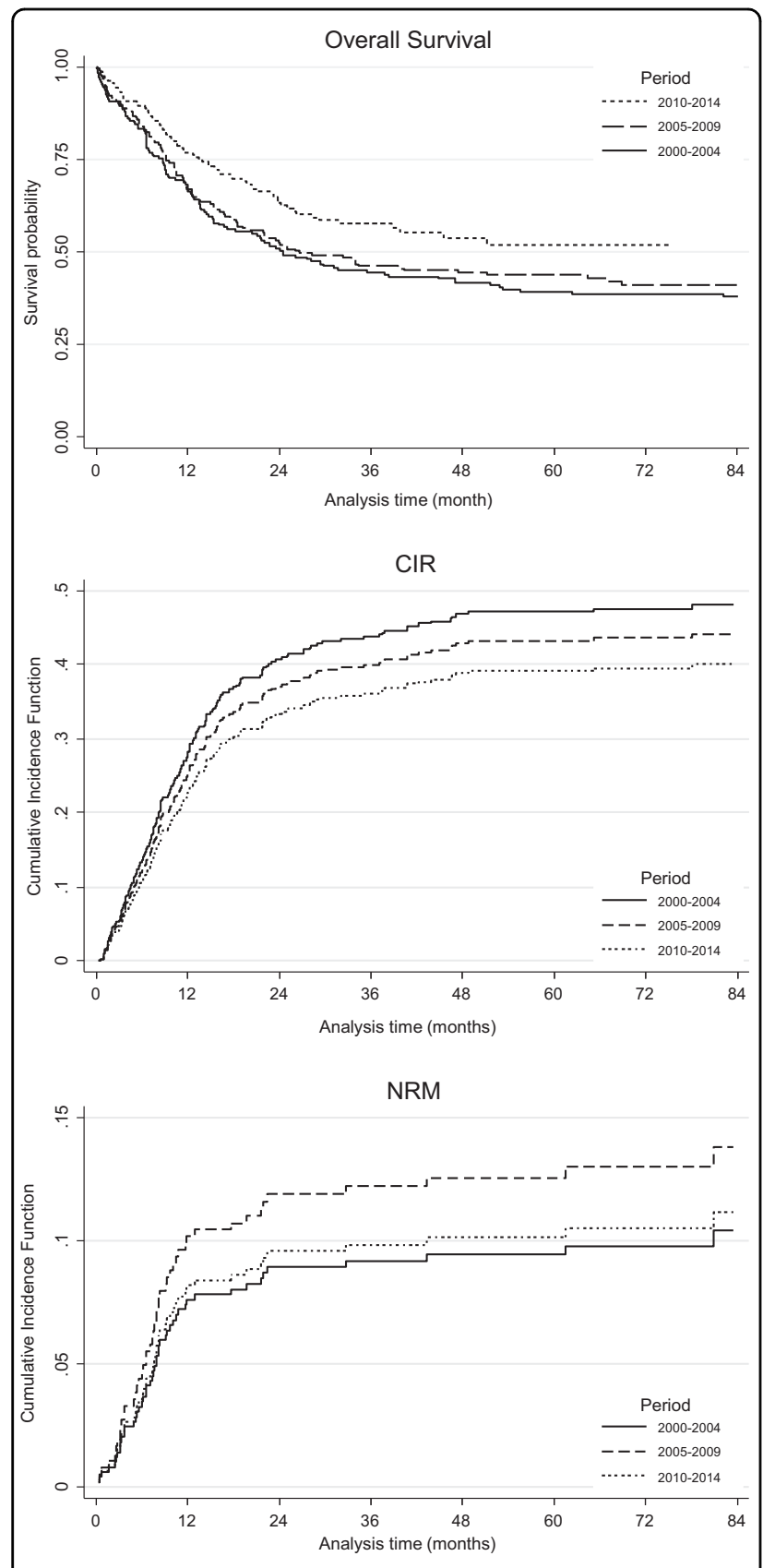

Fig. 1 Overall survival (OS), cumulative incidence of relapse (CIR) and non-relapse mortality (NRM) in first complete response in younger (<60 years) AML patients

therapeutic course) could be critical for the activity of anthracyclines. Furthermore, we and other have previously shown that a $60 \mathrm{mg} / \mathrm{m}^{2}$ dose of daunorubicin was equivalent to $90 \mathrm{mg} / \mathrm{m}^{2}$ but inferior to idarubicin ${ }^{13,30,31}$. Whether idarubicin $\left(45 \mathrm{mg} / \mathrm{m}^{2}\right)$ is better than daunorubicin $\left(270 \mathrm{mg} / \mathrm{m}^{2}\right)$ during induction only remains to be determined and is currently tested in a phase 3 trial of the ALFA/FILO French intergroup (Backbone InterGroup-1 trial, BIG-1, ClinicalTrials.gov: NCT02416388). The
Table 3 Multivariate analysis for overall survival in younger ( $<60$ years) AML patients

\begin{tabular}{llll}
\hline & HR & $\mathbf{9 5 \%} \mathbf{C l}$ & $\mathbf{P}$ \\
\hline 2005-2009 & 0.94 & $0.72-1.24$ & 0.676 \\
2010-2014 & 0.66 & $0.49-0.91$ & 0.011 \\
Age $\geq 50$ years & 1.27 & $1.00-1.62$ & 0.057 \\
Secondary AML & 1.91 & $1.43-2.57$ & $<0.001$ \\
Cytogenetic risk & & & \\
Intermediate & 3.09 & $1.83-5.21$ & $<0.001$ \\
Adverse & 5.37 & $3.04-9.47$ & $<0.001$ \\
WBC > 50 giga/L & 1.58 & $1.18-2.10$ & 0.002 \\
Allo-SCT in CR1 & 0.66 & $0.49-0.89$ & 0.006 \\
\hline
\end{tabular}

Cl confidence interval, CR1 first complete response, HR hazard ratio, SCT stem cell transplantation, WBC white blood cell count

Table 4 Outcome of older ( $\geq 60$ years) AML patients

\begin{tabular}{lllll}
\hline & $\begin{array}{l}\mathbf{2 0 0 0 - 2 0 0 4} \\
\mathbf{N = 1 3 3}\end{array}$ & $\begin{array}{l}\mathbf{2 0 0 5 - 2 0 0 9} \\
\mathbf{N = 1 6 3}\end{array}$ & $\begin{array}{l}\mathbf{2 0 1 0 - 2 0 1 4} \\
\mathbf{N}=\mathbf{1 6 7}\end{array}$ & $\mathbf{P}$ \\
\hline Day-60 death, $n(\%)$ & $21(15.8)$ & $27(16.6)$ & $17(10.2)$ & 0.196 \\
CR1, n (\%) & $89(66.9)$ & $107(65.6)$ & $125(74.9)$ & 0.150 \\
Induction failure, n (\%) & $23(17.3)$ & $30(18.4)$ & $23(13.8)$ & 0.498 \\
Allo-SCT in CR1, n (\%) & 0 & $7(6.5)$ & $24(19.2)$ & $<0.001$ \\
Auto-SCT in CR1, n (\%) & 0 & $2(1.9)$ & 0 & 0.187 \\
CR2, n (\%) & $10 / 60^{\mathrm{a}}(16.7)$ & $12 / 70^{\mathrm{a}}(17.1)$ & $13 / 65^{\mathrm{a}}(20)$ & 0.868 \\
Allo-SCT in CR2 & 0 & 0 & $5(38.5)$ & 0.007 \\
Clinical trial, $n$ (\%) & $51(38.3)$ & $38(23.3)$ & $54(32.3)$ & 0.018 \\
Median DFS, months & $14.3(5.1-52)$ & $14.2(6.7-44)$ & $12.7(6.3-\mathrm{NR})$ & 0.945 \\
(IQR) & & & & \\
Median OS, months & $12.1(3.3-36)$ & $12.3(3.8-39)$ & $16.1(5.9-52)$ & 0.256 \\
(IQR) & & & & \\
\hline
\end{tabular}

$C R 1$ first complete response, $C R 2$ second complete response, DFS disease-free survival, IQR interquartile range, OS overall survival, SCT stem cell transplantation a Relapses after CR1

cumulative dose of cytarabine slightly increased over time together with the decrease of autologous transplantations. It is noteworthy that all therapeutic regimens used in this study included a sufficient cytarabine dose according to the new ELN guidelines ${ }^{32,} 33$. In 2010-2014, we have reduced the doses of cytarabine from 3 to $1.5 \mathrm{~g} / \mathrm{m}^{2}$ only in patients of 50-60 years of age according to a MRC study showing equivalence between the two doses in term of disease control and survival ${ }^{34}$. The BIG-1 trial currently randomizes these two cytarabine doses during post remission therapy to confirm the MRC study in patients $18-60$ years of age. 
Table 5 Multivariate analysis for complete response in older ( $\geq 60$ years) AML patients

\begin{tabular}{|c|c|c|c|}
\hline & OR & $95 \% \mathrm{Cl}$ & $\mathbf{P}$ \\
\hline \multicolumn{4}{|c|}{ Patients with $W B C \leq 50$ giga/L } \\
\hline $2005-2009$ & 0.81 & $0.44-1.48$ & 0.485 \\
\hline 2010-2014 & 0.84 & $0.45-1.54$ & 0.568 \\
\hline Age $\geq 70$ years & 0.68 & $0.43-1.05$ & 0.085 \\
\hline Secondary AML & 0.42 & $0.26-0.68$ & $<0.001$ \\
\hline \multicolumn{4}{|l|}{ Cytogenetic risk } \\
\hline Intermediate & 0.30 & $0.06-1.36$ & 0.117 \\
\hline Adverse & 0.14 & $0.03-0.66$ & 0.013 \\
\hline \multicolumn{4}{|c|}{ Patients with $W B C>50$ giga/L } \\
\hline $2005-2009$ & 0.58 & $0.21-1.59$ & 0.292 \\
\hline 2010-2014 & 3.90 & $1.30-1.7$ & 0.015 \\
\hline Age $\geq 70$ years & 0.68 & $0.43-1.05$ & 0.085 \\
\hline Secondary AML & 0.42 & $0.26-0.68$ & $<0.001$ \\
\hline \multicolumn{4}{|l|}{ Cytogenetic risk } \\
\hline Intermediate & 0.30 & $0.06-1.36$ & 0.117 \\
\hline Adverse & 0.14 & $0.03-0.66$ & 0.013 \\
\hline
\end{tabular}

$C l$ confidence interval, $O R$ odds ratio, $W B C$ white blood cell count

Despite early intensification of anthracycline during induction, the early death rate decreased over time in both younger and older patients. This is likely due to a better management of chemotherapy-induced aplasia rather than a better disease control since the induction failure rate did not change. New antifungal therapies are of high interest in this context and posaconazole, which has been approved for primary fungal infection prophylaxis during induction, remains one of the rare drugs to be associated with an improvement of survival in AML patients treated by intensive chemotherapy ${ }^{23}$. Moreover, there has been a recent reduction of mortality due to invasive aspergillosis during induction in $\mathrm{AML}^{35}$.

A major progress observed in the recent period, concerned patients with hyperleucocytosis. This was especially the case for older patients in whom a significant period effect was found for both response and OS. Since 2010, we use dexamethasone in patients with high WBC count. The rationale was to target leukostasis and inflammatory dysregulations often observed in those patients $^{36}$. We have shown that the addition of dexamethasone to intensive chemotherapy was associated with a significant improvement of both disease-free and OS in hyperleukocytic AML patients ${ }^{24}$. Moreover, very recent preclinical studies have shown that the development of cytarabine resistance is associated with increased sensitivity to glucocorticoids indicating that our clinical
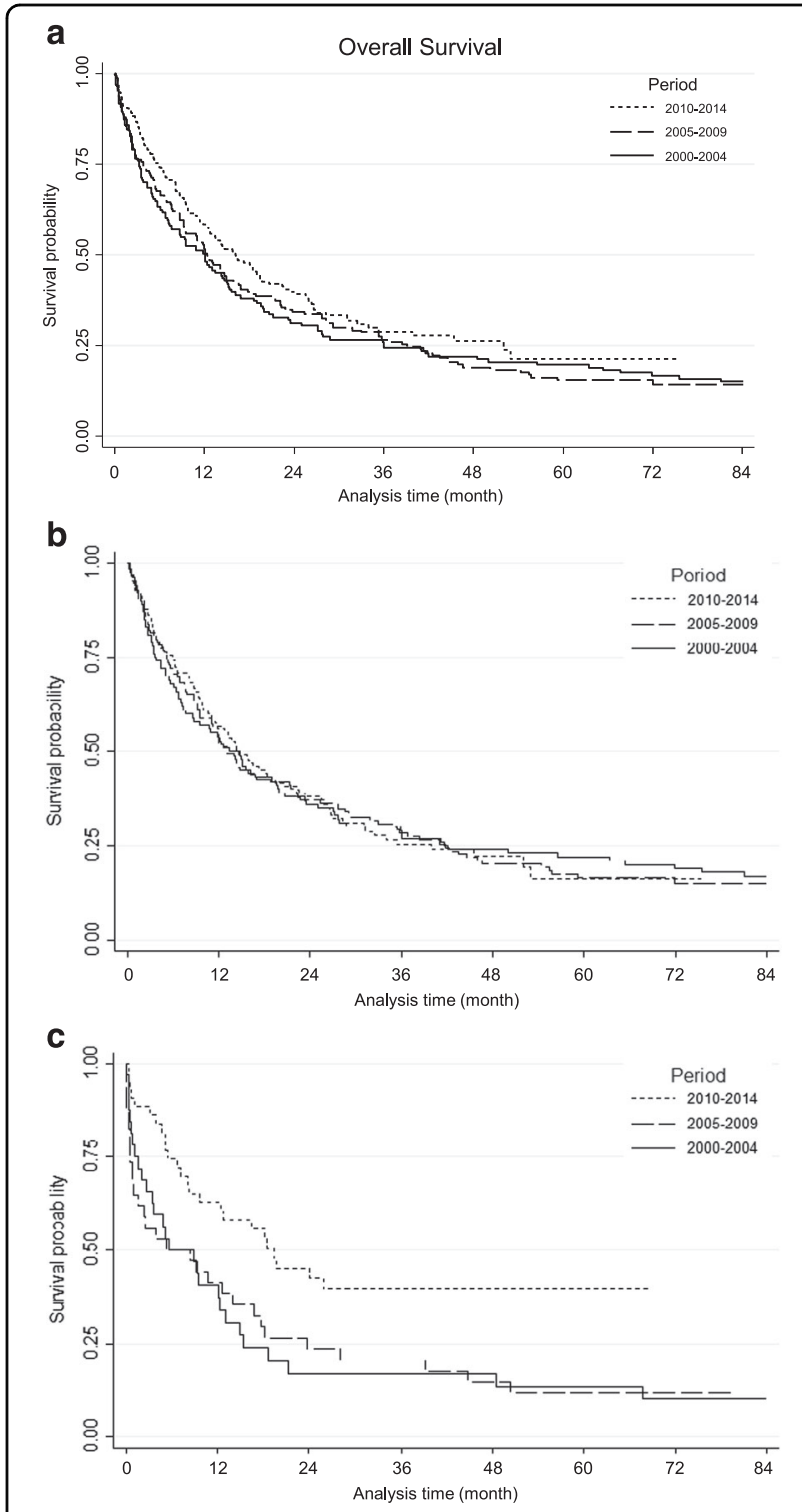

Fig. 2 a Overall survival in the whole cohort of AML patients $\geq 60$ years b in patients $\geq 60$ years with $W B C \leq 50$ giga/L $\mathbf{c}$ and in patients $\geq 60$ years with $W B C>50$ giga/L

results may suggest a synergistic effect of dexamethasone with chemotherapy, at least in hyperleukocytic patients ${ }^{24}$, 37, 38 .

Autologous stem cell transplantation in first CR is still advocated by some investigators and included as a therapeutic option in the ELN guidelines ${ }^{39}$. It is suggested that autologous transplantation could benefit to favorable or intermediate-risk AML patients especially those with negative minimal residual disease. We have progressively abandoned this strategy in first CR along with molecular stratification and extension of allogeneic stem cell transplantation indications (i.e., favorable risk treated by cytarabine; others allocated to allogeneic transplantation 
Table 6 Multivariate analysis for overall survival in older ( $\geq 60$ years) AML patients

\begin{tabular}{lccl}
\hline & HR & $\mathbf{9 5 \%} \mathbf{C l}$ & $\mathbf{P}$ \\
\hline Patients with WBC $\leq$ 50 giga/L & & \\
2005-2009 & 1.11 & $0.83-1.48$ & 0.497 \\
2010-2014 & 1.17 & $0.87-1.59$ & 0.304 \\
Age $\geq 70$ years & 1.36 & $1.09-1.69$ & 0.006 \\
Secondary AML & 1.53 & $1.20-1.94$ & 0.001 \\
Cytogenetic risk & & & \\
Intermediate & 2.60 & $1.28-5.27$ & 0.008 \\
Adverse & 4.74 & $2.28-9.89$ & $<0.001$ \\
Patients with WBC $>50$ giga/L & & \\
2005-2009 & 1.05 & $0.62-1.76$ & 0.861 \\
2010-2014 & 0.41 & $0.24-0.71$ & 0.002 \\
Age $\geq 70$ years & 1.36 & $1.09-1.69$ & 0.006 \\
Secondary AML & 1.53 & $1.20-1.94$ & 0.001 \\
Cytogenetic risk & & & \\
Intermediate & 2.60 & $1.28-5.27$ & 0.008 \\
Adverse & 4.74 & $2.28-9.89$ & $<0.001$ \\
\hline
\end{tabular}

Cl confidence interval, $H R$ hazard ratio, WBC white blood cell count

${ }^{a}$ Allogeneic stem cell transplantation in first complete response was not independently associated with overall survival in older ( $\geq 60$ years) AML patients

whenever possible). Although, our strategy was associated with a better survival in the 2010-2014 period, we acknowledge that the exact role of autologous transplantation remains to be prospectively reassessed in selected patients with time-dependent statistical analyses ${ }^{40}$.

The main limitation of our study is its retrospective design. Our database is prospectively annotated since 2007 with follow-up for all patients since our center is the sole accredited for induction chemotherapy and allogeneic stem cell transplantation in our Region ${ }^{41}$. It is also possible that some of our routine therapeutic management may not be applied in other centers.

Over time, we have optimized the chemotherapy's backbone, the management of adverse events or specific situations, such as hyperleucocytosis and extended the indications to allogeneic stem cell transplantation. It will be challenging to further improve OS of AML patients with current therapeutic options. We hope that the next major improvements will be achieved in a near future with the arrival of new potent drugs with different mechanism of action ${ }^{42}$.

\section{Acknowledgements}

We thank the data management unit of Toulouse University for his support enabling e-CRF. We thank all the members of the G.A.E.L (Gaël Adolescent Espoir
Leucémie) association. This study was supported by grants from the French government under the "Investissement d'avenir" program (ANR-11-PHUC-001)

\section{Author details}

'Service d'Hématologie, Centre Hospitalier Universitaire de Toulouse, Institut Universitaire du Cancer de Toulouse Oncopole, Toulouse, France. ${ }^{2}$ Université Toulouse III Paul Sabatier, Toulouse, France. ${ }^{3}$ Cancer Research Center of Toulouse, UMR1037-INSERM, ERL5294 CNRS, Toulouse, France. ${ }^{4}$ Laboratoire d'Hématologie, Centre Hospitalier Universitaire de Toulouse, Institut Universitaire du Cancer de Toulouse Oncopole, Toulouse, France. ${ }^{5}$ Service d'Epidémiologie, Centre Hospitalier Universitaire de Toulouse, Toulouse, France. ${ }^{6}$ UMR 1027, INSERM-Université de Toulouse III, Toulouse, France

\section{Competing interests}

The authors declare that they have no competing interests.

\section{Publisher's note}

Springer Nature remains neutral with regard to jurisdictional claims in published maps and institutional affiliations.

\section{Supplementary information}

The online version of this article (https://doi.org/10.1038/s41408-017-0011-1) contains supplementary material.

Received: 4 August 2017 Revised: 12 September 2017 Accepted: 15 September 2017

Published online: 29 November 2017

\section{References}

1. DeAngelo D. J., Stein E. M. \& Ravandi F. Evolving therapies in acute myeloid leukemia: progress at last?Am. Soc. Clin. Oncol. Educ. Book 35, e302-e312 (2016).

2. Sekeres M. A. \& Steensma D. P. Boulevard of broken dreams: drug approval for older adults with acute myeloid leukemia. J. Clin. Oncol. 30, 4061-4063 (2012).

3. Dohner H., Weisdorf D. J. \& Bloomfield C. D. Acute myeloid leukemia. N. Engl. J. Med. 373, 1136-1152 (2015).

4. Ellison, L. F. Increasing survival from leukemia among adolescents and adults in Canada: a closer look. Health Rep. 27, 19-26 (2016).

5. Sant, M. et al. Survival for haematological malignancies in Europe between 1997 and 2008 by region and age: results of EUROCARE-5, a population-based study. Lancet Oncol. 15, 931-942 (2014).

6. Thein, M. S., Ershler, W. B., Jemal, A., Yates, J. W. \& Baer, M. R. Outcome of older patients with acute myeloid leukemia: an analysis of SEER data over 3 decades. Cancer 119, 2720-2727 (2013).

7. Derolf, A. R. et al. Improved patient survival for acute myeloid leukemia: a population-based study of 9729 patients diagnosed in Sweden between 1973 and 2005. Blood 113, 3666-3672 (2009).

8. Pulte, D., Gondos, A. \& Brenner, H. Improvements in survival of adults diagnosed with acute myeloblastic leukemia in the early 21st century. Haematologica 93, 594-600 (2008).

9. Grimwade, D. et al. Refinement of cytogenetic classification in acute myeloid leukemia: determination of prognostic significance of rare recurring chromosomal abnormalities among 5876 younger adult patients treated in the United Kingdom Medical Research Council trials. Blood 116, 354-365 (2010).

10. LaRochelle, O. et al. Do AML patients with DNMT3A exon 23 mutations benefit from idarubicin as compared to daunorubicin? A single center experience. Oncotarget 2, 850-861 (2011).

11. Thomas, X. et al. Randomized phase II study of clofarabine-based consolidation for younger adults with acute myeloid leukemia in first remission. J. Clin. Oncol. 35, 1223-1230 (2017).

12. Pigneux, A. et al. Addition of androgens improves survival in elderly patients with acute myeloid leukemia: a GOELAMS study. J. Clin. Oncol. 35, 387-393 (2017).

13. Recher, C. et al. Long-term results of a randomized phase 3 trial comparing idarubicin and daunorubicin in younger patients with acute myeloid leukaemia. Leukemia 28, 440-443 (2014). 
14. Jourdan, E. et al. Prospective evaluation of gene mutations and minimal residual disease in patients with core binding factor acute myeloid leukemia. Blood 121, 2213-2223 (2013).

15. Lioure, B. et al. Early matched sibling hematopoietic cell transplantation for adult AML in first remission using an age-adapted strategy: long-term results of a prospective GOELAMS study. Blood 119, 2943-2948 (2012).

16. Pigneux, A. et al. Addition of lomustine to idarubicin and cytarabine improves the outcome of elderly patients with de novo acute myeloid leukemia: a report from the GOELAMS. J. Clin. Oncol. 28, 3028-3034 (2010).

17. Dohner, $\mathrm{H}$. et al. Diagnosis and management of acute myeloid leukemia in adults: recommendations from an international expert panel, on behalf of the European LeukemiaNet. Blood 115, 453-474 (2010).

18. Bacigalupo, A. et al. Defining the intensity of conditioning regimens: working definitions. Biol. Blood Marrow Transplant. 15, 1628-1633 (2009).

19. Chevallier, $P$. et al. Tandem versus single autologous peripheral blood stem cell transplantation as post-remission therapy in adult acute myeloid leukemia patients under 60 in first complete remission: results of the multicenter prospective phase III GOELAMS LAM-2001 trial. Leukemia 24, 1380-1385 (2010)

20. Fine, J. \& G, R. A proportional hazards model for the subdistribution of a competing risk. J. Am. Stat. Assoc. 94, 496-509 (1999).

21. Bories, P. et al. Intensive chemotherapy, azacitidine, or supportive care in older acute myeloid leukemia patients: an analysis from a regional healthcare network. Am. J. Hematol. 89, E244-E252 (2014).

22. Chabrol, A. et al. Prophylaxis of invasive aspergillosis with voriconazole or caspofungin during building work in patients with acute leukemia. Haematologica 95, 996-1003 (2010).

23. Cornely, O. A. et al. Posaconazole vs. fluconazole or itraconazole prophylaxis in patients with neutropenia. N. Engl. J. Med. 356, 348-359 (2007).

24. Christian Récher, S. B. et al. Dexamethasone reduces incidence of relapse and improves overall survival in Hyperleucocytic acute myeloid leukemia. Blood 128, 1636 (2016).

25. Schlenk, R. F. et al. Mutations and treatment outcome in cytogenetically normal acute myeloid leukemia. N. Engl. J. Med. 358, 1909-1918 (2008).

26. Cornelissen, J. J. et al. Results of a HOVON/SAKK donor versus no-donor analysis of myeloablative HLA-identical sibling stem cell transplantation in first remission acute myeloid leukemia in young and middle-aged adults: benefits for whom? Blood 109, 3658-3666 (2007).

27. Koreth, J. et al. Allogeneic stem cell transplantation for acute myeloid leukemia in first complete remission: systematic review and meta-analysis of prospective clinical trials. JAMA 301, 2349-2361 (2009).
28. Fernandez, H. F. et al. Anthracycline dose intensification in acute myeloid leukemia. N. Engl. J. Med. 361, 1249-1259 (2009).

29. Schaich, M. et al. High-dose cytarabine consolidation with or without additional amsacrine and mitoxantrone in acute myeloid leukemia: results of the prospective randomized AML2003 trial. J. Clin. Oncol. 31, 2094-2102 (2013).

30. Devillier, R. et al. Comparison of 60 or $90 \mathrm{mg} / \mathrm{m}(2)$ of daunorubicin in induction therapy for acute myeloid leukemia with intermediate or unfavorable cytogenetics. Am. J. Hematol. 90, E29-E30 (2015).

31. Burnett, A. K. et al. A randomized comparison of daunorubicin $90 \mathrm{mg} / \mathrm{m} 2$ vs $60 \mathrm{mg} / \mathrm{m} 2$ in AML induction: results from the UK NCRI AML17 trial in 1206 patients. Blood 125, 3878-3885 (2015).

32. Dohner, $\mathrm{H}$. et al. Diagnosis and management of AML in adults: 2017 ELN recommendations from an international expert panel. Blood 129, 424-447 (2017).

33. Lowenberg, B. Sense and nonsense of high-dose cytarabine for acute myeloid leukemia. Blood 121, 26-28 (2013).

34. Burnett, A. K. et al. Optimization of chemotherapy for younger patients with acute myeloid leukemia: results of the medical research council AML15 trial. J. Clin. Oncol. 31, 3360-3368 (2013)

35. Dragonetti, G., Criscuolo, M., Fianchi, L. \& Pagano, L. Invasive aspergillosis in acute myeloid leukemia: are we making progress in reducing mortality? Med. Mycol. 55, 82-86 (2017).

36. Stucki, A., Rivier, A. S., Gikic, M., Monai, N., Schapira, M. \& Spertini, O. Endothelia cell activation by myeloblasts: molecular mechanisms of leukostasis and leukemic cell dissemination. Blood 97, 2121-2129 (2001).

37. Kurata, M. et al. Using genome-wide CRISPR library screening with library resistant DCK to find new sources of Ara-C drug resistance in AML. Sci. Rep. $\mathbf{6}$, 36199 (2016).

38. Malani D. et al. Enhanced sensitivity to glucocorticoids in cytarabine-resistant AML. Leukemia 31, 1187-1195 (2016).

39. Zuckerman, T., Beyar-Katz, O. \& Rowe, J. M. Should autotransplantation in acute myeloid leukemia in first complete remission be revisited? Curr. Opin. Hematol 23, 88-94 (2016).

40. Gorin, N. C. et al. Autologous stem cell transplantation for adult acute leukemia in 2015: time to rethink? Present status and future prospects. Bone Marrow Transplant. 50, 1495-1502 (2015).

41. Bertoli, S. et al. Time from diagnosis to intensive chemotherapy initiation does not adversely impact the outcome of patients with acute myeloid leukemia. Blood 121, 2618-2626 (2013).

42. Stein, E. M. \& Tallman, M. S. Emerging therapeutic drugs for AML. Blood 127 71-78 (2016). 